\title{
Satisfacción de calidad de servicio en los estudiantes de las Facultades de Ingeniería de la Universidad Nacional Mayor de San Marcos, Perú. Una mirada a través del modelo SERVQUAL
}

\section{Satisfaction of quality of service in the students of the engineering faculties of the Universidad Nacional Mayor of San Marcos, Peru.} A look through the SERVQUAL model.

\author{
Jorge Vergiú Canto ${ }^{1}$ (i)
}

Recibido: 15-01-2021; Aceptado: 25-04-2021; Publicado: 28-04-2021.

\section{RESUMEN}

El objetivo de esta investigación fue diagnosticar la satisfacción de los Estudiantes con relación a la calidad de los servicios, en las Facultades de Ingeniería de la Universidad Nacional Mayor de San Marcos, Perú, sustentado en el modelo de calidad de servicio Servqual de Parasuman, Zeithaml y Berry (1985). Se abordó desde una ontología realista, una epistemología positivista y un enfoque cuantitativo. Los resultados reflejan un nivel bajo en la satisfacción de los Estudiantes, al ubicarse los 4 indicadores que miden la variable con un Índice de Calidad de Servicio (ICS) en menos 1: Enseñanza y Aprovechamiento (Fiabilidad) en -0,48; Gestión del Bienestar del Estudiante (Capacidad de Respuesta) en -0.46, Gestión de la Infraestructura de Ambientes de Clase (Elementos Tangibles) en -0.66, Habilidades Blandas (Empatía) en -0.15; obteniéndose un ICG de -1.74. Se concluye que la Universidad objeto de estudio, como un sector generador de beneficio social, debe emprender acciones estrategias que permitan elevar el ICS para satisfacer las expectativas de sus Estudiantes y convertirse en agente de cambio y de alta competitividad.

Palabras clave: Satisfacción de expectativas; índice de calidad de servicio; Modelo de Calidad de Servicio Servqual; calidad; fiabilidad; capacidad de respuesta; elementos tangibles; empatía.

\section{ABSTRACT}

The objective of this research was to diagnose the satisfaction of the Students in relation to the quality of the services, in the Faculties of Engineering of the Universidad Nacional Mayor de San Marcos, Peru, based on the quality of service model Servqual de Parasuman, Zeithaml and Berry (1985). It was approached from a realistic ontology, a positivist epistemology and a quantitative 
approach. The results reflect a low level in the satisfaction of the Students, when the 4 indicators that measure the variable are located with a Service Quality Index (ICS) at minus 1: Teaching and Achievement (Reliability) at -0.48; Student Wellbeing Management (Responsiveness) at -0.46 , Classroom Infrastructure Management (Tangible Elements) at -0.66, Soft Skills (Empathy) at -0.15 ; obtaining an ICG of -1.74 . It is concluded that the University under study, as a generating sector of social benefit, must undertake strategic actions that allow raising the ICS to meet the expectations of its Students and become an agent of change and high competitiveness.

Keywords: Satisfaction of expectations; service quality index; Servqual Service Quality Model; quality; reliability; answer's capacity; tangible items; empathy.

\section{INTRODUCCIÓN}

Las Instituciones de Educación Superior tienen grandes retos ante la crisis producida por el Covid-19. Así lo planteó el director del Instituto Internacional para la Educación Superior en América Latina y el Caribe (IESALC), Francesc Pedró, quien resaltó, entre otros, estos retos: (a) la conexión tecnológica aseverando que apenas el 51\% de los viviendas de América Latina disponen de los equipos necesarios para conectarse a Internet; y (b) problemas emocionales de los Estudiantes, puesto que las 2/3 partes de los Estudiantes en el ámbito mundial refieren tener problemas de estrés, angustia, nervios, ira, ansiedad, rabia, impotencia, debido a lo que ha significado la autorregulación de los procesos de aprendizaje (IESALC, 2020). Esta problemática hace que la Educación, como sector estratégico, juegue un papel fundamental para el logro de la competitividad de cualquier país, por lo que debe emprender acciones que le permitan evaluar su calidad continuamente y determinar hasta qué punto está logrando satisfacer a sus Estudiantes para que se inserten en la sociedad como agentes de cambio.

En el caso de Perú, así como en otros países de América Latina, se hace necesario la sincronización del Sistema Educativo con los otros sectores de la economía, ya que se requiere de la formación de individuos con alto nivel de desempeño para que pueda ser un país competitivo en el ámbito nacional e internacional. Más, si se considera que en el Ranking de Competitividad Mundial, elaborado por el Institute of Management Development (IMD) de Suiza, en asociación con Centrum Pucp para el desarrollo del capítulo de Perú, se ubicó en el número 52 de 63 países, ranking que mide la capacidad que tiene cada nación de generar prosperidad al usar todos los recursos disponibles y la competencia de su economía (Centrum Pucp, 2020). En este sentido, este ranking se convierte en una herramienta potente que ayuda a determinar los puntos claves para seguir mejorando competitivamente en los años próximos. Con respecto a los resultados generales, los primeros lugares del ranking son ocupados, en orden, por Singapur, Dinamarca, Suiza, Holanda y Hong Kong, constituyéndose estos países en ejemplos a seguir en la implementación de estrategias competitivas y buenas prácticas, dado sus notables desempeños en los indicadores evaluados.

Por lo consiguiente, Perú tiene un gran desafío, como es el de ubicarse en un mejor puesto de competitividad, evaluando los factores que están incidiendo en ese resultado. $Y$ uno de ellos es la calidad del Sector Educativo, que está siendo más afectada después de la pandemia. Datos revelados de la investigación de Figallo, González y Diestra (2020), plantean que (a): cerca de 650 mil Estudiantes en el ámbito nacional dejarán de lado sus estudios, de los cuales trescientos cincuenta mil de universidades privadas y trescientos mil de institutos; (b) la realización de significativas inversiones no previstas para implementar la Educación en Línea, principalmente en equipos, software y servicios de comunicación, para poner en marcha las actividades de Teletrabajo para todos los Trabajadores y Docentes, ha afectado el presupuesto de las Instituciones Educativas, sumándole, además, el apoyo que han tenido que dar a Estudiantes de bajos recursos para dotarlos de equipo y mejorar su conectividad; (c) se han visto reducidos exorbitantemente los ingresos de rentas y servicios debido a la deserción (se calcula entre 
Vergiú Canto - Satisfacción de calidad de servicio en estudiantes de la Universidad Nacional Mayor de San Marcos, Perú.

$13 \%$ y $18 \%) ;(d)$ se ha producido un recorte de los presupuestos en las áreas académicas y administrativas; siendo menos afectada el área de Investigación, más si están vinculadas a aportar soluciones para enfrentar la pandemia; (e) se han producido, por parte de los Padres de Familia y Estudiantes, reclamos de la calidad de la Educación recibida en la Modalidad Virtual, tanto en los Centros Educativos como en la Superintendencia Nacional de Educación Superior Universitaria (SUNEDU), entidad adscrita al Ministerio de Educación.

No obstante, antes de la pandemia, según datos del Sistema Nacional de Evaluación, Acreditación y Certificación de la Calidad Educativa (SINEACE, 2017), ciento cuarenta y dos (142) universidades, públicas y privadas, han ido implementando nuevos modelos de gestión que buscan la eficiencia de los procesos y la optimización de los recursos. Por otro lado, las universidades públicas miden la eficiencia a través de la calidad de sus procesos con el fin de mejorar las actividades académicas y administrativas.

En la Figura 1, se muestra el plan del SINEACE (2017) con respecto a la relación de los factores de modelamiento en la Acreditación para los Programas de Estudios, donde se observa que la Institución Educativa posee tres pilares: Gestión estratégica, Formación Integral y el Soporte Institucional, observándose que la gestión del Aseguramiento de la Calidad es un eje estratégico, convirtiéndose en indicador de Evaluación Continua y Permanente.

Ahora bien, en el marco de la nueva Ley Universitaria se plantea cambiar el statu quo de la calidad en los servicios para lograr una eficiencia en los procesos operativos involucrados. Para ello, se debe evaluar y hacerle un seguimiento a las expectativas y percepciones de los usuarios, para poderles brindar el bien y servicio que requieren.

\section{GESTIÓN ESTRATÉGICA}

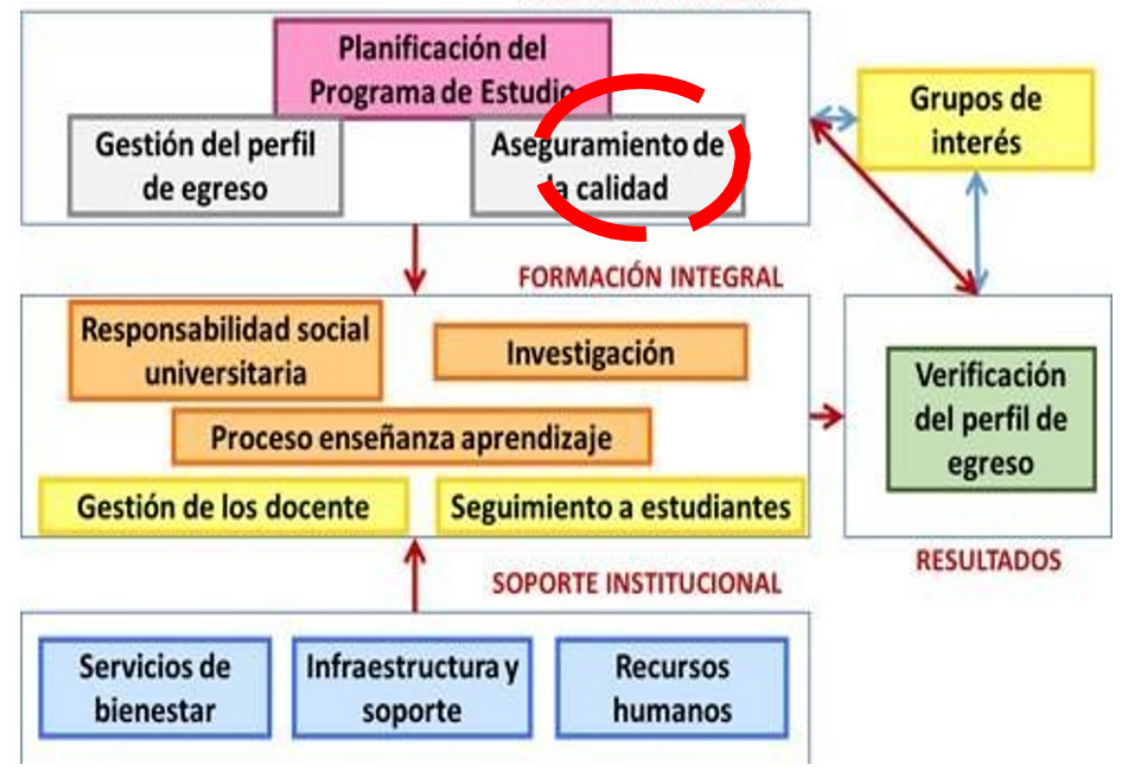

Figura 1. Niveles de posibles expectativas del cliente. Relación de dimensiones y factores y modelos de Acreditación de Programas de Estudios universitarios. Fuente: SINEACE (2017).

A nivel local, la Universidad Nacional Mayor de San Marcos posee una serie de problemas en cuanto a la calidad esperada de sus procesos administrativos y académicos, lo cual es fundamental, pues constituye el soporte y garantía para alcanzar los objetivos que se persiguen en las especialidades de Ingeniería, así como llevar con eficiencia los procedimientos expresados 
en el documento de gestión denominado Reglamento de Organización y Funciones (ROF) que tiene toda la Universidad, pero en la condición de cada Facultad, el Centro de Producción tiene su propio Cuadro de Asignación de Personal (CAP), Manual de Organización y Funciones (MOF) y Manual de Procesos y Procedimientos (MAPRO). Estos problemas se han manifestado por la carencia de personal con las capacidades necesarias para contribuir con los objetivos estratégicos de la Universidad, debido a que la organización no visualiza un área de la gestión de calidad de sus operaciones, adecuada a los requerimientos de la Institución.

Desde esa perspectiva, se hace imprescindible que la Universidad cuente con la ejecución de un adecuado modelo de diagnóstico y evaluación de la Gestión de la Calidad, orientado a la mejora de satisfacción de sus Estudiantes, para cumplir con de las necesidades de calidad en Gestión Pública y ubicarse con excelencia como la principal Universidad del país.

En resumen, el sector universitario en el Perú enfrenta una serie de desafíos y dificultades, como producto del entorno cambiante, la globalización y el ideal de posicionamiento en la sociedad, y, en lo particular, existen factores que afectan a lo interno, entre otros, la falta de una adecuada gestión institucional, reflejada en la poca ejecución presupuestal, ineficiencia en los procesos, deficiencia en los servicios brindados, servicios de mantenimiento deficientes, reflejándose de esa manera la necesidad de lograr una gestión de operaciones eficiente. Por tal motivo, y por las ideas que sustentan la problemática esbozada, se hace necesario realizar esta investigación que tiene como objetivo diagnosticar la satisfacción de los Estudiantes con relación a la calidad de los servicios de las Facultades de Ingeniería de la Universidad Nacional Mayor de San Marcos, Perú.

\section{SUSTENTACIÓN TEÓRICA}

Fundamentos Teóricos de la Calidad. Según Denton (1991), la calidad constituye un conjunto de propiedades de un producto o servicio, que se logra con la implementación de un sistema de producción y distribución, que tenga como objetivo fundamental la satisfacción de las necesidades y expectativas del cliente como principal grupo de interés, al considerar que toda organización, para ser sostenible en el tiempo, debe ser capaz de desarrollar productos y servicios que cumplan con las especificaciones que los clientes demandan.

Feigenbaum (1986) expone que el concepto de calidad expresa un sistema eficiente y eficaz con el objetivo de lograr la integración de todos propósitos de mejora de la gestión, de los distintos grupos de interés de la organización, para proporcionar bienes y servicios a grados que reconozcan la satisfacción del cliente, a un menor costo económico para la empresa.

De igual manera, Crosby (1987), considera que, como el cliente es el principal elemento dentro de una organización, esta debe producir con cero defectos los productos que elabora. Un aspecto importante sobre el Enfoque de Calidad es el hecho de que debe estar en perfecta sincronía con el Plan Estratégico en función del logro de los objetivos empresariales. Un elemento que agrega Deming (1989) al proceso de Gestión de la Calidad es el ciclo PDCA (por sus siglas en inglés Plan- Do- Check- Act), el gerente debe planear, desarrollar, comprobar y actuar, para poder lograr grados predecibles de uniformidad y fiabilidad a bajo coste, adecuado a las necesidades del mercado.

Otro concepto lo tiene Juran (1993), quien define los Sistemas de Calidad como la percepción y satisfacción de las expectativas de los clientes, siendo una característica la capacidad de satisfacción de las necesidades de los clientes; además, calidad enfatiza en la capacidad de no tener deficiencias, lo que denominó la adecuación para el uso satisfaciendo las necesidades del cliente. 
El autor, Puig-Duran (2006), menciona que "la calidad del servicio es un concepto abstracto y complejo, difícil de definir y medir" (p. 4). Sin embargo, Parasuraman, Zeithahaml y Berry (1988) mencionan que "la idea de conceptualizar la calidad del servicio se encuentra en la percepción de los propios clientes" (p.1). Plantean Ganga, Alarcón y Pedraja (2019, p.4) que "la percepción del usuario o cliente hace referencia a como este estima que la organización cumple con la entrega del servicio, de acuerdo con la manera como él valora lo que recibe". Y esto va a estar influenciado por sus expectativas, es decir, de lo que se espera sea el servicio que recibe de la organización. Para Drucker (1990), la percepción de la calidad es lo que el usuario obtiene del bien o servicio y no es lo que se pone dentro de estos, pues expresa la manera como ellos sienten al recibirlo para la satisfacción de sus necesidades. Según Parasuraman, Zeithmal y Berry (1988), definen la percepción como la amplitud de la discrepancia o diferencia que exista entre las expectativas o deseos de los usuarios

Como se ha planteado, la mayoría de las definiciones de calidad de servicios, están centradas en cubrir las necesidades y los requerimientos de los consumidores y la manera de cómo estos servicios prestados alcanzan o superan sus expectativas. Siendo así, se puede constatar la existencia de dos conceptos en la ecuación de la calidad del servicio: por un lado, las expectativas; y, por otro, las percepciones: Expectativas - Percepciones, de la cual, se desprende el servicio esperado desde la perspectiva del cliente, tal como se visualiza en la Figura 2.

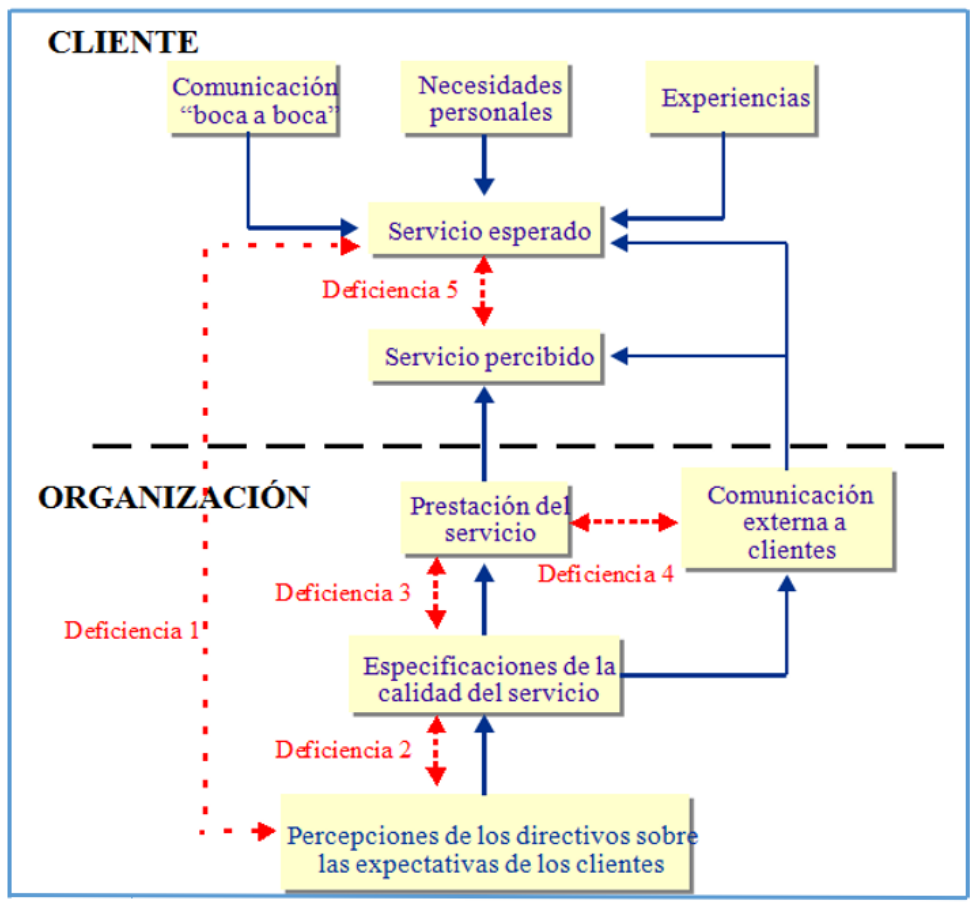

Figura 2. Modelo de calidad de servicio (teórico). Fuente: Parasuman, Zeithaml y Berry, 1985).

Metodología SERVQUAL. De la teoría mostrada anteriormente, se desarrolla una adaptación al Modelo de Calidad de Servicio de la Metodología Servqual (Ver Figura 2). Parasuman, et al (1985) definieron la calidad de servicio como deferencia entre la calidad esperada y la percibida. A esta diferencia le denominaron Gap 5 (Diferencia 5) y este Gap a su vez depende de otras cuatro brechas según muestran las líneas punteadas de la Figura 2. Todas estas brechas ayudan a identificar y medir las eficiencias o ineficiencias en la gestión de los servicios. 
En la Figura 3 se muestra la aplicación del modelo de calidad de servicio que será desarrollado en la presente investigación.

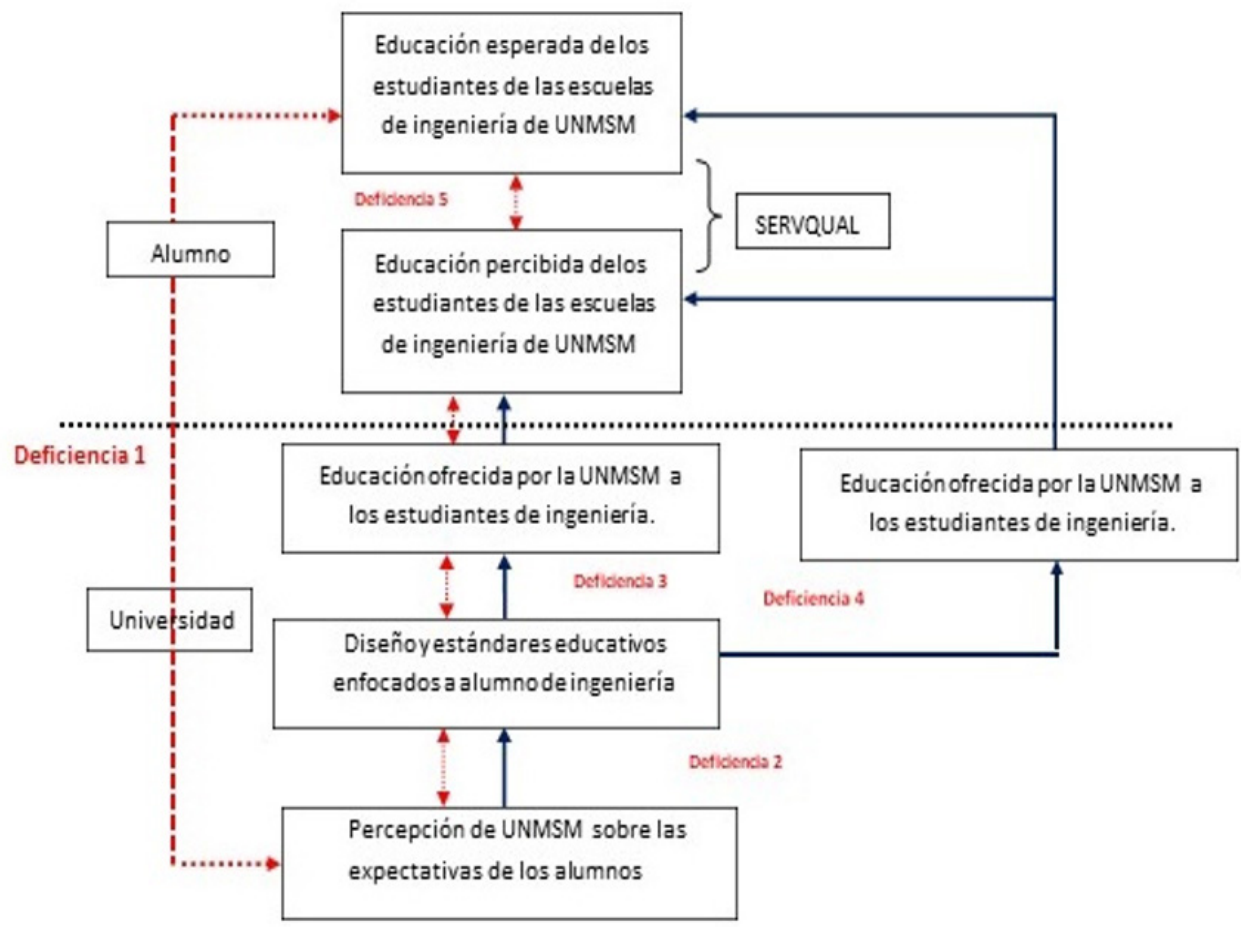

Figura 3. Modelo de calidad de servicio (teórico) aplicado a la UNMSM. Fuente: Adaptado de Parasuman, Zeithaml \& Berry (1985) y por el investigador.

El Modelo Servqual establece cuatro indicadores que permiten medir la calidad de los servicios de la Universidad objeto de estudio (Zeithaml, Bitner y Gremler, 2009):

Fiabilidad. Se refiere a la habilidad para ejecutar el servicio prometido de forma fiable y cuidadosa. En este caso, se medirá lo referente al proceso de enseñanza y aprovechamiento de la Universidad objeto de estudio.

Capacidad de respuesta o sensibilidad. Es la disposición para ayudar a los usuarios y para prestarles un servicio rápido y adecuado. Se refiere a la atención y prontitud al tratar las solicitudes, responder preguntas y quejas de los clientes y solucionar problemas. En esta investigación se diagnóstica, con este indicador, la gestión del bienestar estudiantil.

Elementos tangibles. Es la apariencia física, instalaciones físicas, como la infraestructura, equipos, materiales, personal. En esta investigación está representada por la gestión de la infraestructura de ambientes de clase.

Empatía. Referida a la atención individualizada que ofrecen las instituciones a sus clientes. Se debe transmitir por medio de un servicio personalizado o adaptado al gusto del cliente. Este indicador mide en esta investigación a las habilidades blandas.

En el Tabla 1, se muestra que la variable objeto de estudio se mide mediante 4 indicadores a través de 21 ítems. 
Vergiú Canto - Satisfacción de calidad de servicio en estudiantes de la Universidad Nacional Mayor de San Marcos, Perú.

Tabla 1. Operacionalización de la variable con sus respectivos ítems.

\begin{tabular}{|c|c|c|}
\hline Variables & Indicadores & Ítems \\
\hline \multirow{5}{*}{ Calidad de servicios } & Habilidades blandas (Empatía) & Del 5 al 9 \\
\hline & Enseñanza y aprovechamiento (Fiabilidad) & Del 10 al 14 \\
\hline & $\begin{array}{l}\text { Gestión del bienestar del Estudiante } \\
\text { (Capacidad de respuesta) }\end{array}$ & Del 15 al 20 \\
\hline & $\begin{array}{c}\text { Gestión de la infraestructura de ambientes de clase } \\
\text { (Elementos tangibles) }\end{array}$ & Del 21 al 25 \\
\hline & Aspectos generales & Del 1 al 4 \\
\hline $\begin{array}{l}\text { Satisfacción de } \\
\text { expectativas }\end{array}$ & \multicolumn{2}{|c|}{$\begin{array}{c}\text { Diferencia entre las expectativas y percepciones de los Estudiantes con relación a la calidad } \\
\text { de los servicios }\end{array}$} \\
\hline
\end{tabular}

\section{ASPECTOS METODOLÓGICOS}

La investigación es de tipo descriptiva, con un diseño transeccional, contemporáneo, no experimental y de campo, sustentada en un enfoque ontológico realista debido a que el objeto de estudio es abordado de manera independiente al sujeto que investiga. Por lo tanto, esa realidad existente en el contexto de la Universidad no fue modificada, ni transformada en el diagnóstico realizado. Dado esta consideración, el enfoque epistemológico es el empírico-inductivo, lo que sustenta que el hecho es la única realidad científica; y la experiencia y la inducción los métodos exclusivos de la ciencia para generar conocimiento, a partir de un proceso lógico del pensamiento, con visión objetiva de lo observado, con un lenguaje numérico-aritmético.

El enfoque metodológico es el cuantitativo porque se examinaron los datos de manera numérica, especialmente en el campo de la estadística.

La población objeto de estudio fueron los Estudiantes de las Facultades de ingeniería de la Universidad Nacional Mayor de San Marcos constituida por 7.943 unidades. El tamaño de muestra (n) seleccionada será usando la técnica de poblaciones finitas a través de la siguiente fórmula:

$$
n=\frac{N \times Z_{a}{ }^{2} \times p \times q}{d^{2} \times(N-1)+Z_{a}{ }^{2} \times p \times q}
$$

Dónde:

$\mathrm{N}=$ tamaño de la población (7.943)

$Z=$ nivel de confianza $(95 \%)$

$\mathrm{p}=$ probabilidad de éxito, o proporción esperada $(0.5)$

$\mathrm{q}=$ probabilidad de fracaso $(0.5)$

$\mathrm{d}=$ precisión (Error máximo admisible en términos de proporción) (0.05) Cálculo del tamaño de la muestra conociendo el tamaño de la población es:

$$
n=\frac{7.943 \times 1,96^{2} \times 0,5 \times 0,5}{0,5^{2} \times(7.943-1)+1,96^{2} \times 0,5 \times 0,5} \quad n=367
$$


A un nivel de confianza del 95\%, considerando una probabilidad de éxito del $50 \%$, y una precisión del 5\%, a un tamaño de la población de 7.943 Estudiantes, se calculan 367 encuestas a realizar, distribuidas como se muestra en la Tabla 2.

Tabla 2. Distribución de la muestra.

\begin{tabular}{|c|c|c|c|c|c|}
\hline Facultad & Escuela Profesional/Especialidad & $\begin{array}{l}\text { Total por } \\
\text { Facultad }\end{array}$ & $\begin{array}{c}\text { Total } \\
\text { EP/ Espec }\end{array}$ & $\begin{array}{c}\text { Total } \\
\text { (Muestra) } \\
\text { EP/ Espec }\end{array}$ & $\begin{array}{l}\text { Total (\%) } \\
\text { EP/ Espec. }\end{array}$ \\
\hline \multirow{3}{*}{$\begin{array}{l}\text { Química e Ingeniería } \\
\text { Química }\end{array}$} & Química & & 427 & \multirow{3}{*}{59} & 20 \\
\hline & Ingeniería Química & 1283 & 578 & & 27 \\
\hline & Ingeniería Agroindustrial & & 278 & & 13 \\
\hline \multirow{6}{*}{$\begin{array}{c}\text { Ingeniería Geológica, } \\
\text { Minera, Metalúrgica y } \\
\text { Geográfica }\end{array}$} & Ingeniería Geológica & \multirow{6}{*}{1740} & 419 & \multirow{6}{*}{80} & 19 \\
\hline & Ingeniería Geográfica & & 320 & & 15 \\
\hline & Ingeniería de Minas & & 245 & & 11 \\
\hline & Ingeniería Metalúrgica & & 388 & & 18 \\
\hline & Ingeniería Civil & & 293 & & 14 \\
\hline & Ingeniería Ambiental & & 75 & & 3 \\
\hline \multirow{3}{*}{ Ingeniería Industrial } & Ingeniería Industrial & \multirow{3}{*}{1679} & 1219 & \multirow{3}{*}{78} & 56 \\
\hline & Ingeniería Textil y Confecciones & & 372 & & 17 \\
\hline & $\begin{array}{l}\text { Ingeniería de Seguridad } \\
\text { y Salud en el Trabajo }\end{array}$ & & 88 & & 4 \\
\hline \multirow{3}{*}{ Ingeniería Electrónica } & Ingeniería Electrónica & & 693 & \multirow{3}{*}{75} & 32 \\
\hline & Ingeniería Eléctrica & 1634 & 557 & & 26 \\
\hline & Ingeniería de Telecomunicaciones & & 384 & & 18 \\
\hline \multirow{3}{*}{$\begin{array}{c}\text { Ingeniería de } \\
\text { Sistemas e Informática }\end{array}$} & Ingeniería de Sistemas & \multirow{2}{*}{1607} & 1113 & \multirow{2}{*}{74} & 51 \\
\hline & Ingeniería de Software & & 494 & & 23 \\
\hline & Total & 7943 & 943 & 367 & 367 \\
\hline
\end{tabular}

Para la selección de la muestra se utilizó el muestreo de tipo probabilístico y sistemático. En relación con la técnica de recolección de datos se utilizó la encuesta y como instrumento cuestionario del Modelo. Para el procesamiento y análisis de datos fue necesario cuantificar la variable del estudio a través de sus cuatro indicadores, estableciendo una escala para la correcta medición de esta: 1 - muy mala, 2 - mala, 3 - ni buena ni mala, 4 - buena y 5 - muy buena.

Cada indicador fue diagnosticado mediante las técnicas estadísticas de la media, moda, mediana; además, se determinó la variación de los diferentes indicadores con medidas como la desviación estándar, varianza, gráficos de tendencias de los indicadores, entre otros relacionados. Las herramientas usadas fueron los softwares Microsoft Office Excel, Microsoft Office Access y, por último, IBM SPSS. 
Vergiú Canto - Satisfacción de calidad de servicio en estudiantes de la Universidad Nacional Mayor de San Marcos, Perú.

Tabla 3. Índice de calidad en el servicio general.

\begin{tabular}{ccccc}
\hline Promedio & $\begin{array}{c}\text { Enseñanza y } \\
\text { aprovechamiento } \\
\text { (Fiabilidad) }\end{array}$ & $\begin{array}{c}\text { Gestión del bienestar } \\
\text { del Estudiante } \\
\text { (Capacidad de } \\
\text { respuesta) }\end{array}$ & $\begin{array}{c}\text { Gestión de la } \\
\text { infraestructura de } \\
\text { ambientes de clase } \\
\text { (Elementos tangibles) }\end{array}$ & $\begin{array}{c}\text { Habilidades blandas } \\
\text { (Empatía) }\end{array}$ \\
\hline Percepciones & 2.90 & 2.97 & 2.68 & 3.32 \\
Expectativas & 3.38 & 3.42 & 3.34 & 3.47 \\
ICS & -0.48 & -0.46 & -0.66 & -0.15 \\
ICS GLOBAL & $\mathbf{- 1 . 7 4}$ & & & \\
\hline
\end{tabular}

\section{RESULTADOS Y DISCUSIÓN}

A manera de introducción, se considera un Estudiante satisfecho cuando el resultado de la percepción ( $P$ ) y la expectativa (E) para la variable objeto de estudio, se manifieste en una diferencia de cero o un valor positivo y, un usuario insatisfecho, cuando la diferencia de un valor es negativa. En la Tabla 3 se muestra, de manera global, el Índice de Calidad de Servicio (ICS) para la satisfacción del Estudiante de ingeniería de la UNMSM.

El valor obtenido de $-1.74(-(0.48+0.46+0.66+0.15))$, señala que las percepciones están por debajo de las expectativas formuladas por los Estudiantes de ingeniería, por lo tanto, se deduce que los Estudiantes reciben menos de lo que esperaban del servicio educativo que se les brinda. Esto coincide con los planteamientos de Zeithahaml, Bitner y Gremler, (2009), al plantear que un cliente está insatisfecho cuando sus expectativas son mayores que sus percepciones.

Para completar el análisis general se presenta la Tabla 4 con los resultados de la aplicación de la encuesta, mostrando la diferencia entre las preguntas por percepción y expectativa para cada indicador.

En Figura 4, se puede ver como se distribuyen los promedios de cada pregunta de expectativa y percepción, por cada elemento de calidad de manera general considerando las dieciséis escuelas de ingeniería. En la mayoría de las preguntas las expectativas son mayores que las percepciones, solo 2 preguntas muestran un Gap (ICS) positivo (pregunta 1.15 ¿Cómo percibe la pertinencia en las clases? que pertenece a al indicador 2: Gestión del bienestar del Estudiante; y la pregunta 1.7, ¿Cómo percibe el profesionalismo del Docente? que pertenece al indicador 4: Habilidades Blandas), se desprende del grafico se tiene una brecha que se tiene que mejorar para aproximarse a la expectativa que reportan los Estudiantes de ingeniería de la Universidad Nacional Mayor de San Marcos..

La distribución de las respuestas a las preguntas del cuestionario por indicador y su respectivo criterio de calidad SERVQUAL, se muestra en la Figura 5 (percepción) y 6 (expectativa)

De acuerdo con la Figura 5 la mayoría de Estudiantes respondió de manera neutral con la pregunta ni buena ni mala, con un promedio de $45.78 \%$, seguido por la respuesta a la pregunta "buena" con un promedio de $24.24 \%$ y para la respuesta "muy buena" el promedio es de $2.34 \%$ haciendo un total de $72.36 \%$ de respuestas positiva con respecto a la percepción de los Estudiantes de ingeniería de la UNMSM, frente a la satisfacción del servicio educativo que se les brinda. 
Tabla 4. Resultados de las Encuestas.

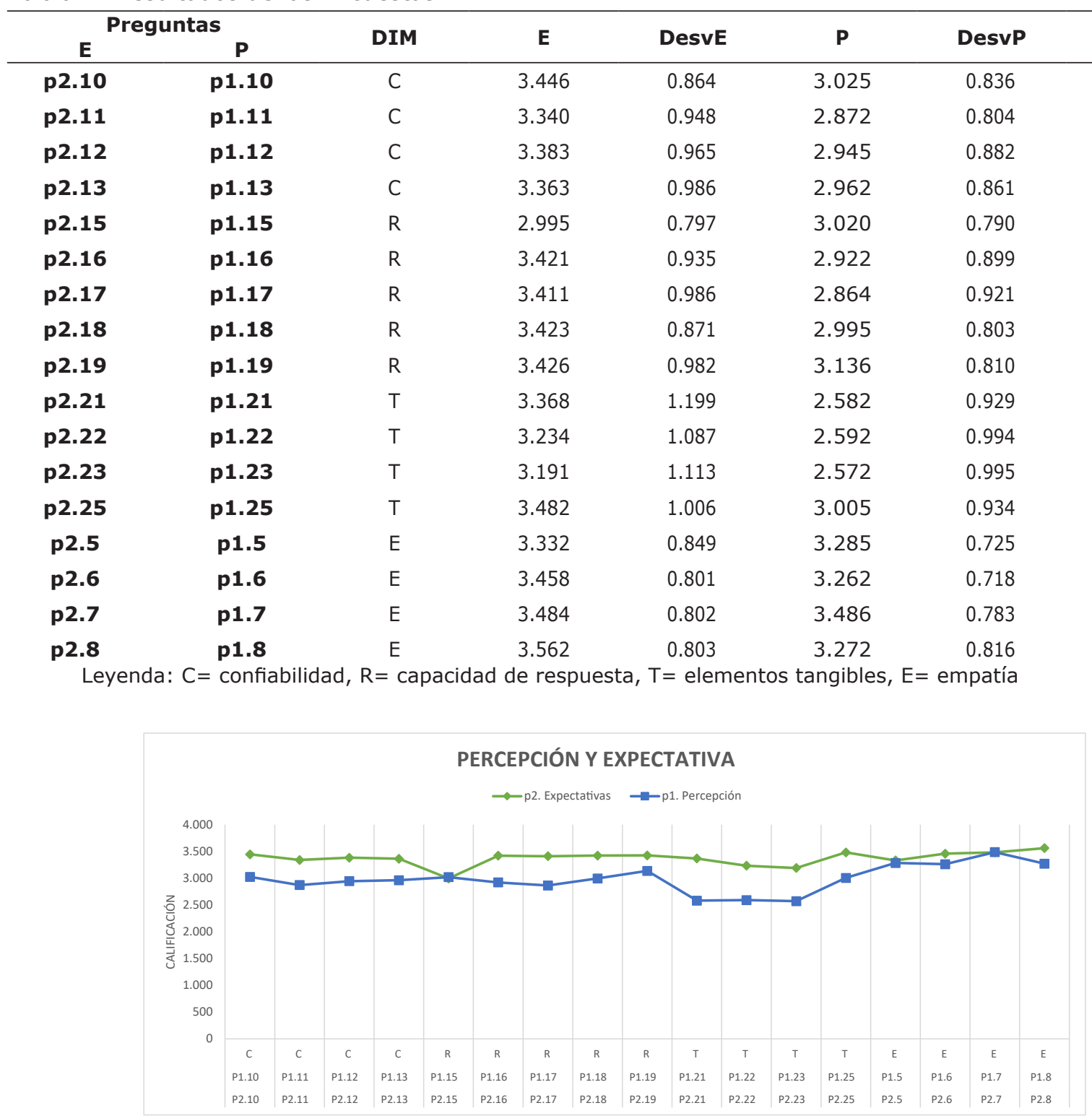

Figura 4. Promedio de cada sección del cuestionario respecto a cada pregunta

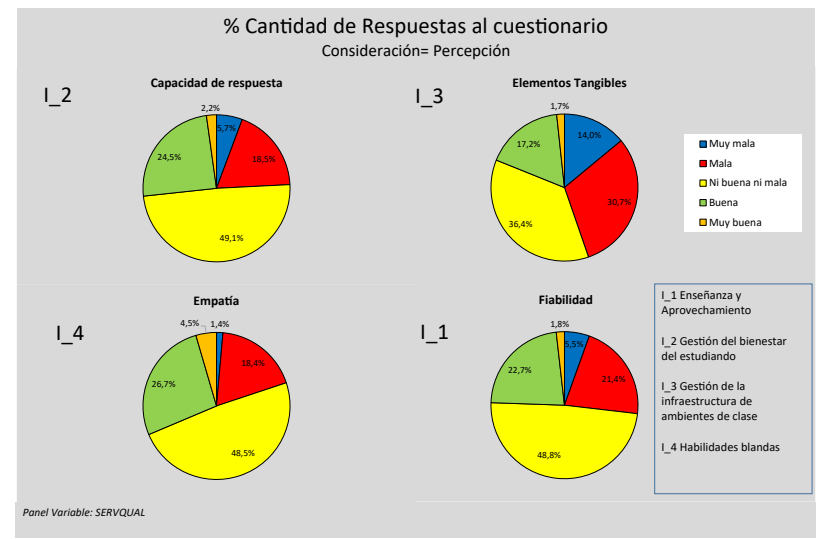

Figura 5. Distribución de respuestas por Criterios de Calidad SERVQUAL (Percepción). 
De acuerdo con la Figura 6 se desprende que la mayoría de Estudiantes ha respondido de manera neutral con la pregunta "buena" con un promedio de $36.18 \%$, seguido por la respuesta a la pregunta "ni buena ni mala" con un promedio de $36.11 \%$ y para la respuesta "muy buena" el promedio es de $10.61 \%$ haciendo un total de $82.90 \%$ de respuestas positiva con respecto a la expectativa de los Estudiantes de ingeniería de la UNMSM, frente a la satisfacción del servicio educativo que se les brinda.

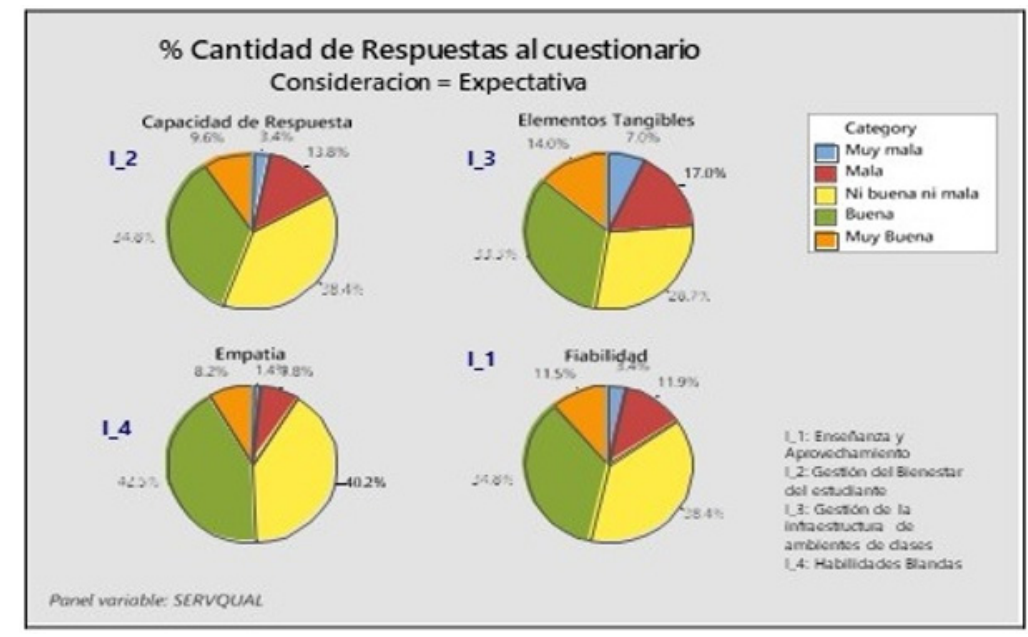

Figura 6. Distribución de respuesta por Criterios de Calidad SERVQUAL (Expectativa)

Al comparar las Figuras 5 y 6 se concluye que la expectativa (E) de los Estudiantes es mayor a la percepción $(P)$ que tiene frente a la satisfacción del servicio $(82.9 \%>72.36 \%)$, con lo cual se comprueba que se tiene una brecha de mejora como se muestra en la Figura 2. Estos resultados coinciden con los alcanzados por Cieza, Castillo, Garay y Poma (2014), en su investigación titulada: satisfacción de los Estudiantes de una Facultad de medicina peruana, cuya muestra fue de 188 Estudiantes, solo el 8\% estaba satisfecho con los cuatros indicadores estudiados.

Distribución de encuestas por las Facultades de Ingeniería. De las cinco (5) Facultades de ingeniería de la UNMSM, las más representativas son en el siguiente orden: Facultad de Ingeniería Industrial con un 20.98\%, Facultad de Ingeniería Electrónica y Eléctrica con 20.71\%., Facultad de Química e Ingeniería Química con 20.44\%, Facultad de Geología, Minas, Metalurgia, Geográfica con $19.07 \%$, Facultad de Ingeniería. Sistemas e Informática con $18.80 \%$, de acuerdo con la Figura 7.

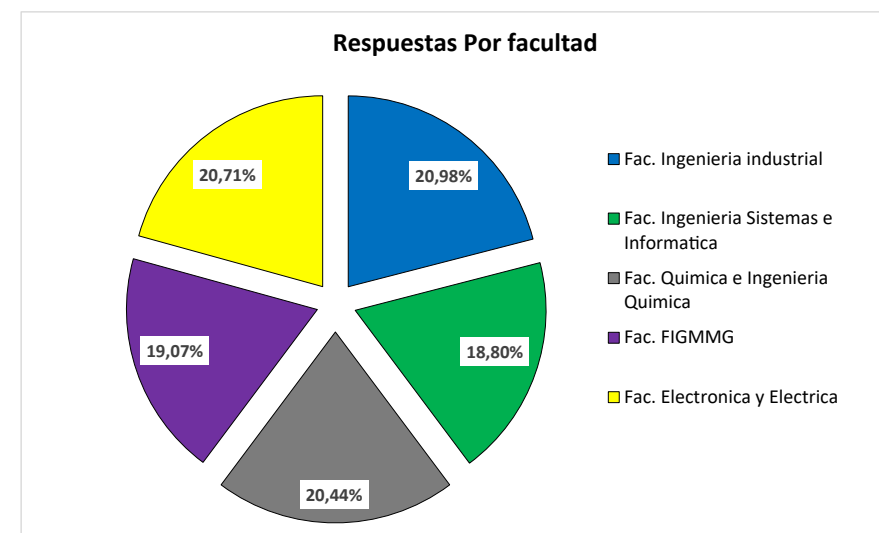

Figura 7. Distribución de respuestas por Facultad de ingeniería. 
Satisfacción de los Estudiantes (Percepción menos expectativas) con relación a la calidad de servicios. Índice de Calidad de Servicio (ICS) por Facultad de ingeniería.

Indicador 1. Enseñanza y Aprovechamiento. En la Tabla 5, se muestra el ISC por Facultad de Ingeniería de la UNMSM, para el indicador: Enseñanza y Aprovechamiento (criterio SERVQUAL: Fiabilidad).

Tabla 5. Percepción, Expectativa e ICS por escuela de ingeniería. Indicador enseñanza y aprovechamiento

\begin{tabular}{cccccc}
\hline \multirow{2}{*}{ Facultad } & $\begin{array}{c}\text { Percepción enseñanza } \\
\text { aprovechamiento }\end{array}$ & $\begin{array}{c}\text { Expectativas ensenanza } \\
\text { aprovechamiento }\end{array}$ & \multicolumn{3}{c}{$\begin{array}{c}\text { Índice de calidad. } \\
\text { Enseñanza y aprovechamiento }\end{array}$} \\
\cline { 2 - 6 } & Media & Media & Media & Desviación típica & Recuento \\
\hline FII & 2,93 & 3,40 & $-0,47$ & 0,92 & 77 \\
FISI & 2,92 & 3,57 & $-0,65$ & 0,90 & 69 \\
FQIQ & 2,88 & 3,61 & $-0,74$ & 0,93 & 75 \\
FIGMMG & 2,79 & 3,17 & $-0,37$ & 1,00 & 70 \\
FIEE & 2,94 & 3,32 & $-0,38$ & 0,93 & 76 \\
\hline
\end{tabular}

De acuerdo con la Figura 8, la Facultad que presenta mayor diferencia en las respuestas son los encuestados de la Facultad de Química e Ingeniería Química (dos escuelas) con ISC de - 0.74, valor que indica que hay una calidad deficiente con respecto a la expectativa, y las demás Facultades como Ingeniería de Sistemas e Informática (dos escuelas) con -0.65, Facultad de Ingeniería Industrial (tres escuelas) -0.47, Facultad de Ingeniería Electrónica y Eléctrica (tres escuelas) con - 0.38, y Facultad de Ingeniería Geológica, Minas, Metalurgia, Geográfica (seis escuelas) con -0.37, que también indican una calidad deficiente con respecto a la expectativa.

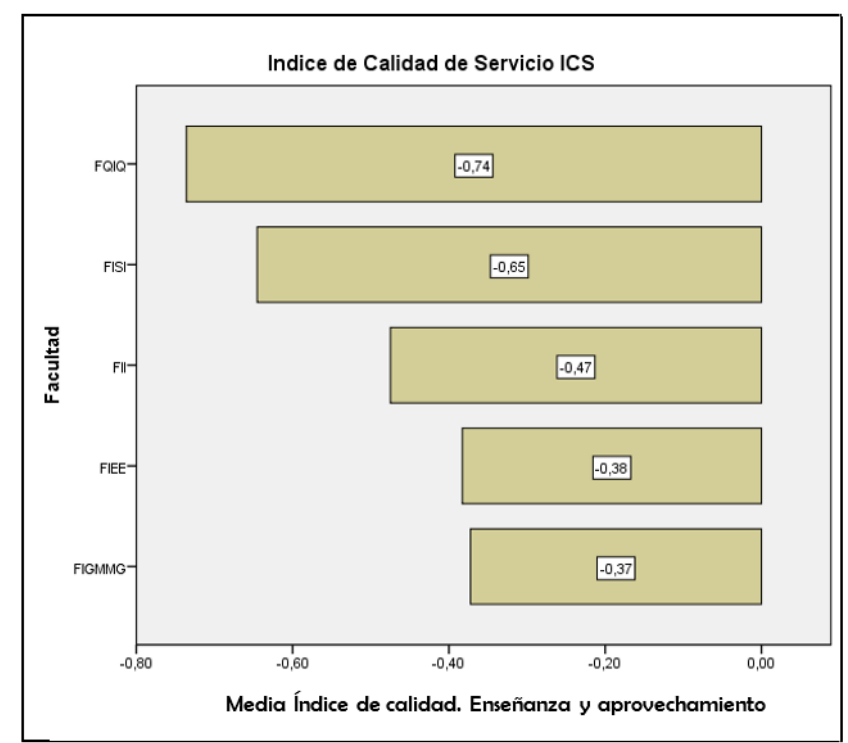

Figura 8. Media ICS por Facultad de Ingeniería. Indicador enseñanza y aprovechamiento. 
Vergiú Canto - Satisfacción de calidad de servicio en estudiantes de la Universidad Nacional Mayor de San Marcos, Perú.

Indicador 2. Gestión del Bienestar del Estudiante. En la Tabla 6, se muestra el ISC por Facultad de Ingeniería de UNMSM, para el indicador: Gestión del Bienestar del Estudiante (criterio SERVQUAL: Capacidad de Respuesta).

Tabla 6. Percepción, Expectativa e ICS por Facultad de Ingeniería. Indicador 2. Gestión del bienestar del Estudiante

\begin{tabular}{cccccc}
\hline \multirow{2}{*}{ Facultad } & $\begin{array}{c}\text { Percepción gestion } \\
\text { bienestar }\end{array}$ & $\begin{array}{c}\text { Expectativas } \\
\text { gestion bienestar }\end{array}$ & \multicolumn{2}{c}{ Gestión del bienestar del estudiante } \\
\cline { 2 - 6 } & Media & Media & Media & Desviación típica & Recuento \\
\hline FII & 2,98 & 3,39 & $-0,41$ & 0,82 & 77 \\
FISI & 3,08 & 3,48 & $-0,40$ & 0,94 & 69 \\
FQIQ & 2,99 & 3,61 & $-0,62$ & 0,90 & 75 \\
FIGMMG & 2,81 & 3,16 & $-0,35$ & 0,87 & 70 \\
FIEE & 2,98 & 3,31 & $-0,33$ & 0,78 & 76 \\
\hline
\end{tabular}

De acuerdo con la Tabla 6 y figura 9, la Facultad que presenta mayor diferencia en las respuestas son los encuestados de la Facultad de Química e Ingeniería Química (dos escuelas) con ISC de 0.62 , valor que indica que hay una calidad deficiente con respecto a la expectativa, y las demás Facultades como Ingeniería de Industrial (tres escuelas) con - 0.41, Facultad de Ingeniería de Sistema e Informática (dos escuelas) -0.40, Facultad de Ingeniería Geológica, Minas, Metalurgia, Geográfica (seis escuelas) con -0.35, Facultad de Ingeniería Electrónica y Eléctrica (tres escuelas) con -0.33 que también indican una calidad deficiente con respecto a la expectativa.

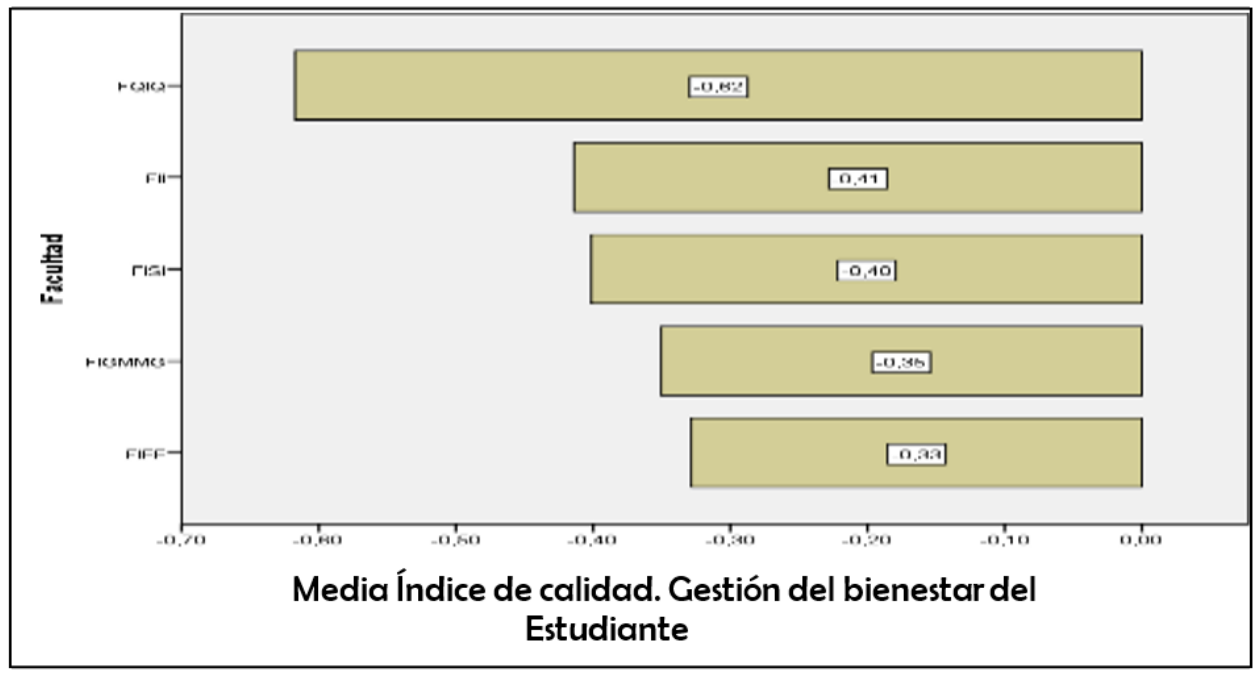

Figura 9. Media Índice de calidad por Facultad. Gestión del bienestar del Estudiante.

Indicador 3. Gestión de la Infraestructura de Ambientes de Clases. En la Tabla 7 se muestra el ISC por Facultad de Ingeniería de la UNMSM, para el indicador: Gestión de la Infraestructura de Ambientes de Clases (criterio SERVQUAL: Elementos Tangibles). 
Tabla 7. Percepción, Expectativa e ICS por Facultad de ingeniería. Indicador: Gestión de la Infraestructura de Ambientes de Clases

\begin{tabular}{cccccc}
\hline \multirow{2}{*}{ Facultad } & $\begin{array}{c}\text { Percepción gestion } \\
\text { infraestructura }\end{array}$ & $\begin{array}{c}\text { Expectativas gestion } \\
\text { infraestructura }\end{array}$ & \multicolumn{3}{c}{$\begin{array}{c}\text { Índice de calidad. } \\
\text { Gestión de la infraestructura de ambiente de } \\
\text { clases }\end{array}$} \\
\cline { 2 - 6 } & Media & Media & Media & Desviación típica & Recuento \\
\hline FII & 2,74 & 3,31 & $-0,57$ & 0,81 & 77 \\
FISI & 2,68 & 3,48 & $-0,81$ & 0,96 & 69 \\
FQIQ & 2,77 & 3,62 & $-0,85$ & 0,93 & 75 \\
FIGMMG & 2,49 & 3,10 & $-0,61$ & 0,90 & 70 \\
FIEE & 2,47 & 3,12 & $-0,65$ & 0,89 & 76 \\
\hline
\end{tabular}

De acuerdo con la Tabla 7 y Figura 10, la Facultad que presenta mayor diferencia en las respuestas son los encuestados de la Facultad de Química e Ingeniería Química (dos escuelas) con ISC de -0.85 , valor que indica que hay una calidad deficiente con respecto a la expectativa, y las demás Facultades como Ingeniería Sistema e Informática (dos escuelas) con -0.81, Facultad de Ingeniería de Electrónica y Eléctrica (tres escuelas) - 0.65, Facultad de Ingeniería Geológica, Minas, Metalurgia, Geográfica (seis escuelas) con -0.61, Facultad de Ingeniería Industrial (tres escuelas) con -0.57 que también indican una calidad deficiente con respecto a la expectativa.

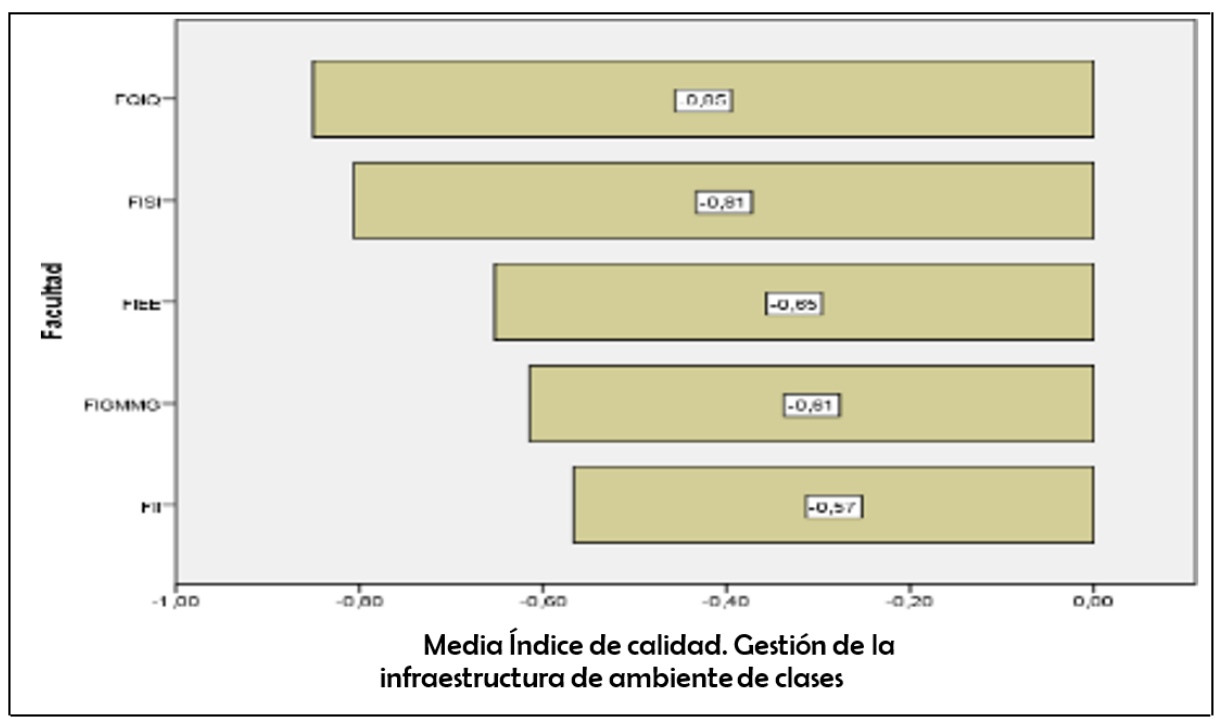

Figura 10. Media Índice de calidad. Gestión de la infraestructura de ambiente de clases.

Indicador 4. Habilidades Blandas. En la Tabla 8 se muestra el ISC por Facultad de Ingeniería de UNMSM, para el indicador: Habilidades Blandas (criterio SERVQUAL: Empatía). 
Vergiú Canto - Satisfacción de calidad de servicio en estudiantes de la Universidad Nacional Mayor de San Marcos, Perú.

Tabla 8. Percepción, Expectativa e ICS por Facultad de ingeniería. Indicador: habilidades blandas

\begin{tabular}{cccccc}
\hline Facultad & $\begin{array}{c}\text { Percepción } \\
\text { habilidades blandas }\end{array}$ & $\begin{array}{c}\text { Expectativas } \\
\text { habilidades blandas }\end{array}$ & \multicolumn{3}{c}{$\begin{array}{c}\text { Índice de calidad. } \\
\text { Habilidades blandas }\end{array}$} \\
\cline { 2 - 6 } Media & Media & Media & Desviación típica & Recuento \\
\hline FII & 3,37 & 3,54 & $-0,17$ & 0,68 & 77 \\
FISI & 3,33 & 3,53 & $-0,21$ & 0,51 & 69 \\
FQIQ & 3,37 & 3,70 & $-0,33$ & 0,55 & 75 \\
FIGMMG & 3,22 & 3,28 & $-0,07$ & 0,60 & 70 \\
FIEE & 3,38 & 3,40 & $-0,20$ & 0,66 & 76 \\
\hline
\end{tabular}

De acuerdo a la Tabla 8 y la Figura 11, la Facultad que presenta mayor diferencia en las respuestas son los encuestados de la Facultad de Química e Ingeniería Química (dos escuelas) con ISC de -0.33 , valor que indica que hay una calidad deficiente con respecto a la expectativa, y las demás Facultades como Ingeniería Sistema e Informática (dos escuelas) con -0.21, Facultad de Ingeniería Industrial (tres escuelas) con -0.17, Facultad de Ingeniería Geológica, Minas, Metalurgia, Geográfica (seis escuelas) con -0.07 Facultad de Ingeniería de Electrónica y Eléctrica (tres escuelas) -0.02 , que también indican una calidad deficiente con respecto a la expectativa.

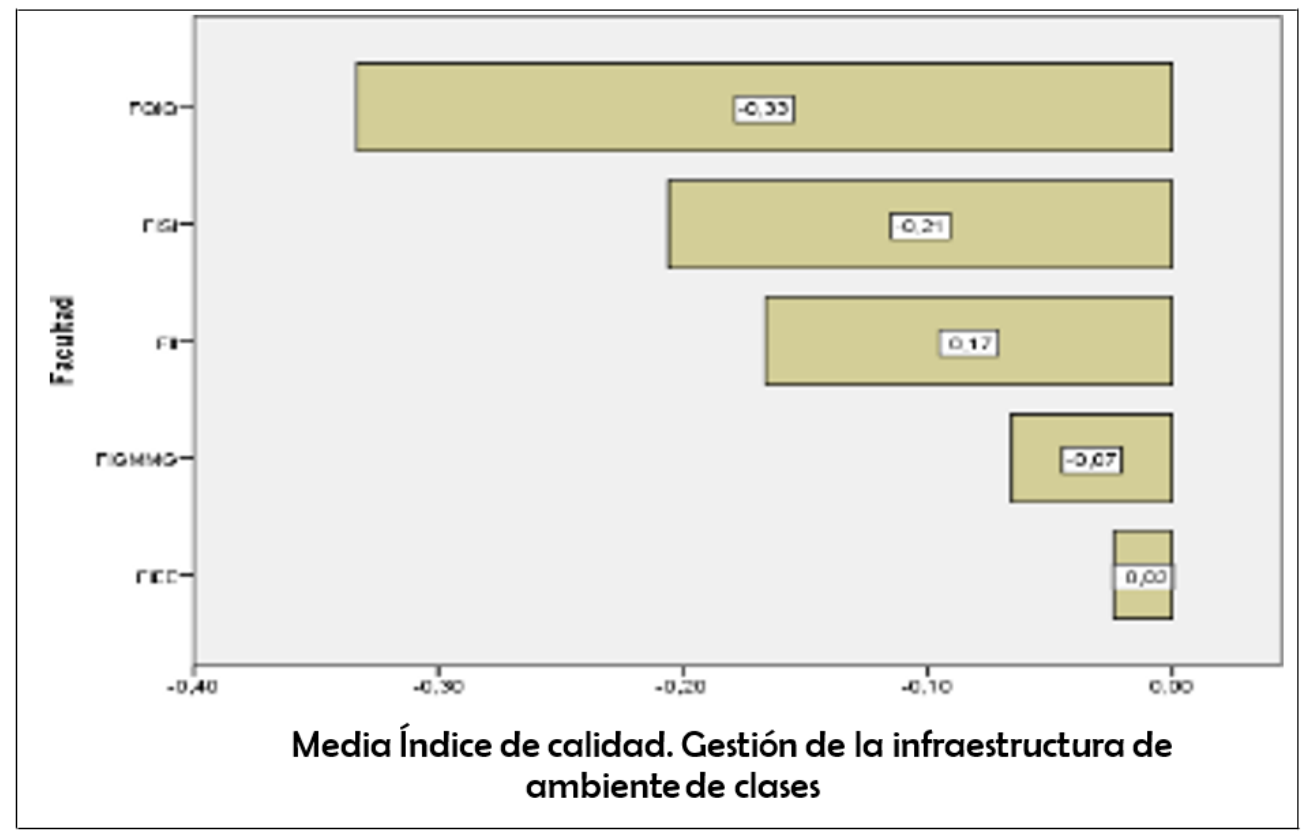

Figura 11. Media Índice de calidad. Habilidades blandas.

Resumen ICS Por Facultad y Por Indicador. De acuerdo con las Tablas 5, 6, 7 y 8, se observa que la exceptiva que tienen los Estudiantes de las escuelas de ingeniería de la UNMSM, es mayor de lo que realmente perciben, con lo cual se tiene una brecha para poder mejorar y acerca de lo que los Estudiantes perciben como satisfacción frente a lo que realmente esperan (expectativas). El resumen se muestra en la Tabla 9. 
Tabla 9. Resumen ICS Por Facultad y Por Indicador.

\begin{tabular}{cccccccc}
\hline \multirow{2}{*}{ Facultad } & ICS_1 & ICS_2 & ICS_3 & ICS_4 & \multicolumn{2}{c}{ ICS_GLOBAL } \\
\cline { 2 - 7 } & Media & Media & Media & Media & Media & Desviación Típica & Recuento \\
\hline FII & $-0,47$ & $-0,41$ & $-0,57$ & $-0,17$ & $-1,62$ & 2,6 & 77 \\
FISI & $-0,65$ & $-0,40$ & $-0,81$ & $-0,21$ & $-2,06$ & 2,72 & 69 \\
FQIQ & $-0,74$ & $-0,62$ & $-0,85$ & $-0,33$ & $-2,54$ & 2,64 & 75 \\
FIGMMG & $-0,37$ & $-0,35$ & $-0,61$ & $-0,07$ & $-1,40$ & 2,48 & 70 \\
FIEE & $-0,38$ & $-0,33$ & $-0,65$ & $-0,20$ & $-1,56$ & 2,57 & 76 \\
\hline
\end{tabular}

Leyenda

\begin{tabular}{cccc}
\hline $\begin{array}{c}\text { Índice de servicio de } \\
\text { calidad (ISC) }\end{array}$ & $\begin{array}{c}\text { Código del } \\
\text { indicador }\end{array}$ & Descripción del indicador & Descripción SERVQUAL \\
\hline ISC_1 & I_1 & $\begin{array}{c}\text { Enseñanza y Aprovechamiento } \\
\text { Gestión del Bienestar del } \\
\text { Estudiante }\end{array}$ & Fiabilidad \\
ISC_2 & I_2 & $\begin{array}{c}\text { Capación de la infraestructura de } \\
\text { ambientes de clases } \\
\text { Habilidades blandas }\end{array}$ & Elementos tangibles \\
ICS_3 & I_3 & Seguridad \\
ICS_4 & I_4 & &
\end{tabular}

De acuerdo a la Tabla 9, la Facultad de que presenta mayor diferencia en las respuestas de los encuestados en la Facultad de Química e Ingeniera Química con ISC_Global de - 2.54 valor que indica que hay una calidad deficiente con respecto a la expectativa, para la Facultad de Ingeniería de Sistemas e Informática con ISC_Global de - 2.06, Facultad de Ingeniera Industrial con ISC_ Global de - 1.62 Facultad de Ingeniera Geologica Minas, Metalurgia, Geografica con ISC_Global de- 1.40, Facultad de Ingeniería Electrónica y Eléctrica con ISC_Global de - 1.39 Considerando todas las escuelas el ISC_Global promedio es -1.80 y comparando con el calculado en la Tabla 3 de -1.74 se tiene una diferencia de -0.06 .

Estos resultados permiten referir lo expuesto por Duque y Chaparro (2012), en cuanto a la necesidad de descubrir y gestionar los componentes de la calidad para el Estudiante, que permitan lealtad de estos para con la Institución de Educación superior, dando prioridad a los procesos medulares.

\section{CONCLUSIONES}

La calidad es un término muy relativo, sin embargo, en lo que todos los autores coinciden es que la calidad se logra cuando una organización logra satisfacer las necesidades de los clientes e incluso superar las expectativas que éstos tienen puestas sobre el producto o servicio que ofrece. Ante esa premisa, la aplicación del clásico Modelo SERVQUAL constituyó una estrategia investigativa que permitió una mejor comprensión en la identificación de los indicadores para medir la calidad del servicio en una Institución Educativa a nivel superior, lo que significa que los resultados obtenidos son fiables y confiables, verificando aún más la pertinencia del Modelo. Por lo tanto, se concluye que la Universidad objeto de estudio, como sector generador de beneficio social, debe emprender acciones estratégicas para mejorar las operaciones en el entorno al soporte institucional enmarcado en la enseñanza y aprovechamiento, la gestión del bienestar del Estudiante, la gestión de la infraestructura de ambientes de clases y las habilidades blandas; que permitan elevar el ICS para satisfacer las expectativas de sus Estudiantes y convertirse en agente de cambio y de alta competitividad. 
Vergiú Canto - Satisfacción de calidad de servicio en estudiantes de la Universidad Nacional Mayor de San Marcos, Perú.

\section{REFERENCIAS}

Centrum Pucp (2020). Resultados del ranking de competitividad 2020. https://cdncentrum.pucp. education/centrum/uploads/2020/06/16160953/informe-ranking-competitividad-2020. pdf?platform=hootsuite

Cieza, J., Castillo, A., Garay, F. y Poma, J. (2014). Satisfacción de los Estudiantes de una Facultad de medicina peruana. Revista Médica Herediana. Vol. 29. No.1. http://www.scielo.org.pe/ scielo.php?pid=S1018-130X2018000100005\&script=sci arttext\&t/ng=en

Crosby, P. (1990). La calidad no cuesta. El arte de cerciorarse de la calidad. México D.F.: Continental.

Deming, E. (1989). Calidad productividad y competitividad: La salida de la crisis. Madrid: Ediciones Díaz de Santos.

Denton, D. (1991). Calidad en el servicio a los clientes. Madrid: Ediciones Díaz de Santos.

Druker, P. (1990). El ejecutivo eficaz. Buenos Aires: Sudamericana.

Duque, E. y Chaparro, C. (2012). Medición de la percepción de la calidad del servicio de Educación por parte de los Estudiantes de la UPTC Duitama. Disponible en: https://www.researchgate. net/publication/254400070 Medicion de la percepcion de la calidad del servicio de educacion por parte de los Estudiantes de la UPTC Duitama

Feigenbaum, V (1986). Control total de la calidad, México: CECSA.

Figallo F., González, M. y Diestra, V. (2020). Perú: Educación superior en el contexto de la pandemia covid 19. Revista de Educación Superior de América Latina. https://rcientificas. uninorte.edu.co/index.php/esal/article/view/13404/214421444832

Ganga, F., Alarcón, N. y Pedraja, L. (2019). Medición de calidad de servicio mediante el modelo SERVQUAL: el caso del Juzgado de Garantía de la ciudad de Puerto Montt Chile. Disponible en: https://www.scielo.cl/scielo.php?script=sci arttext\&pid=S071833052019000400668\&lang $=p t$

Gronroos, C. (1994). Marketing y Gestión de Servicios. Madrid: Ediciones Díaz de Santos.

Instituto Internacional de la UNESCO para la Educación Superior en América Latina y el Caribe (IESALC) (2020). El día después: retos de la Educación superior ante la nueva normalidad. https://www.iesalc.unesco.org/2020/11/11/el-dia-despues-retos-de-la-educacion-superiorante-la-nueva-normalidad/

Juran, J. (1993). Juran on planning for quality. New York: The Free cross-funcional.

Ministerio de Educación (2014). Ley universitaria 30220. http://www.minedu.gob.pe/reformauniversitaria/pdf/ley universitaria.pdf

Oh, H. Service quality, customer satisfaction, and customer value: A holistic Perspective. 
Revista International Journal of Hospitality Management. Vol. 18. No.1. https://www. researchgate.net/publication/222608492 Service quality customer satisfaction and customer value A holistic Perspective

Orellana, A. (2011). Gerencia Universitaria. Estilos Gerenciales y su incidencia en el desempeño Organizacional. El caso de la Universidad de San Carlos de Guatemala. San José Costa Rica: Universidad Estatal a Distancia San José. Costa Rica.

Olorunniwo, F., Hsu, M. y Udo, G. (2006). Service quality, Customer Satisfaction, and behavioral intentions in the service Factory. Revista de marketing de servicios. Vol. 20. No.1. https:// www.researchgate.net/publication/235287046 Service quality Customer Satisfaction and behavioral intentions in the service factory

Parasuraman, A., Zeithahaml, V., y Berry, L. (1985). A conceptual model of service Quality and its implications for future research. New York: Journal Marketing.

Parasuraman, A., Zeithahaml, V., y Berry, L. (1988). SERVQUAL: A multiple-item scale for measuring consumer perceptions of service quality. https://books.google.com.co/books?h $\underline{l=e s \& \mid r=\& i d=p G I P E A A A Q B A J \& o i=f n d \& p g=P A 30 \& o t s=w h g S C r K W G Y \& s i g=d d I x O 0 i C-K V N I F-}$ $\underline{7 z s L Z V l e 6 d B 0 \# v=o n e p a g e \& q \& f=\text { false }}$

Puig- Duran, D. (2006). Fundamentos teóricos de la calidad del servicio. Madrid: Ediciones Díaz de Santos.

Sineace (2017). Modelo de Acreditación para programas Modelo de Acreditación de estudios de Educación superior universitaria. https://fad.unsa.edu.pe/bancayseguros/wp-content/ uploads/sites/4/2019/12/6-Libro-Modelo-de-Acreditaci\%C3\%B3n-para-Programa...-WEB. $\underline{\text { pdf }}$

Zeithahaml, V., Bitner, M., y Gremler, J. (2009). Marketing de servicios. México: Mc Graw Hill. Quinta edición. 